\title{
NEOPLASIA ESCAMOSA DE LA SUPERFICIE OCULAR EN PACIENTES CON PTERIGIÓN EN PERÚ
}

\author{
Luis Furuya-Kanamori1,a, Carlos M. Dulanto-Reinoso 2,3,b, Jennifer C. Stone ${ }^{1, c}$, Lelia Marroquínn $2, b$, \\ Victor Ch. Dulanto-Reinoso ${ }^{4, b}$, José A. Roca ${ }^{5, b}$, Francisco Contreras ${ }^{3,5, b}$, Graham A. Lee ${ }^{1,6,7, a}$
}

\section{RESUMEN}

Objetivos. Estimar la frecuencia de neoplasia escamosa de la superficie ocular (NESO) no sospechada en pterigión, la precisión del diagnóstico clínico y las características demográficas y clínicas asociadas. Materiales y métodos. Se examinaron los informes histopatológicos de los pacientes con diagnóstico clínico de pterigión y/o NESO que fueron quirúrgicamente tratados entre marzo de 2009 y diciembre de 2012 en el Instituto Nacional de Oftalmología en Lima, Perú. La precisión del diagnóstico clínico para identificar la NESO se evaluó mediante la sensibilidad, especificidad y los cocientes de probabilidad. Se realizaron modelos de regresión log-log negativos para identificar las características demográficas y clínicas asociadas con un aumento de las probabilidades de diagnosticar NESO. Resultados. Se examinaron 3021 informes de histopatología. La frecuencia de NESO no sospechada en pterigión fue de 0,65\%. El diagnóstico clínico presentó una sensibilidad del $85 \%$, una especificidad del $99 \%$, un cociente de probabilidad positiva de 111,89 y un cociente probabilidad negativa de 0,15 . Las características asociadas fueron el sexo masculino (OR 1,15; IC 95\%:1,01-1,30), pacientes de 61 a 80 años (OR 1,54; IC 95\%: 1,28-1,85), $\geq$ de 81 años (OR 3,10; IC 95\%: 2,09-4,58), pacientes con lesiones recurrentes (OR 1,59; IC 95\%: 1,03-2,46) y lesiones en el lado temporal (OR 3,57; IC 95\%: 2,63-4,85) presentaron mayor probabilidad de NESO. Conclusiones. Se encontró una baja frecuencia de NESO no sospechada, sin embargo, es recomendable realizar el estudio histopatológico de forma rutinaria para evitar diagnósticos erróneos de NESO como pterigión.

Palabras clave: Pterigion; Neoplasias oculares; Diagnóstico clínico (fuente: DeCS BIREME).

\section{SQUAMOUS NEOPLASIA OF THE OCULAR SURFACE IN PATIENTS WITH PTERYGIUM IN PERU}

\begin{abstract}
Objectives. To estimate the frequency of unsuspected ocular surface squamous neoplasia (OSSN) in pterygium, the accuracy of clinical diagnosis, and associated demographic and clinical characteristics. Materials and methods. We reviewed histopathological reports of patients with a clinical diagnosis of pterygium and/or OSSN who were surgically treated between March 2009 and December 2012 at the National Eye Institute in Lima, Peru. The accuracy of the clinical diagnosis of OSSN was assessed by sensitivity, specificity, and likelihood ratios. Models of negative log-log regression were performed to identify demographic and clinical characteristics associated with increased odds of diagnosing OSSN. Results. 3,021 histopathological reports were reviewed. The frequency of unsuspected OSSN in pterygium was $0.65 \%$. Clinical diagnosis had a sensitivity of $85 \%$, a specificity of $99 \%$, a positive likelihood ratio of 111.89 , and a negative likelihood ratio of 0.15 . Associated characteristics were male gender $(\mathrm{OR}=1.15 ; 95 \% \mathrm{Cl}: 1.01$ to 1.30$)$, age group of 61 80 years $(\mathrm{OR}=1.54,95 \% \mathrm{Cl}: 1.28$ to 1.85$) \geq 81$ years $(\mathrm{OR}=3.10 ; 95 \% \mathrm{Cl}: 2.09$ to 4.58$)$, presence of recurrent lesions $(\mathrm{OR}=1.59 ; 95 \% \mathrm{Cl}: 1.03$ to 2.46$)$ and temporal location lesions (OR $=3.57 ; 95 \% \mathrm{Cl}: 2.63$ to 4.85$)$. These characteristics were associated with a greater likelihood of OSSN. Conclusions. A low frequency of unsuspected OSSN was found; however, it is recommended to routinely perform histopathology studies to avoid misdiagnosis of OSSN as pterygium.
\end{abstract}

Key words: Pterygium; Eye neoplasms; Clinical diagnosis (source: MeSH, NLM).

\footnotetext{
The University of Queensland. Brisbane, Australia

Instituto Nacional de Oftalmología. Lima, Perú

Universidad Peruana Cayetano Heredia. Lima, Perú

Universidad de de Sao Paulo. Sao Paulo, Brasil

Servicio de Oftalmología, Clínica Ricardo Palma. Lima, Perú

City Eye Centre. Brisbane, Australia

Royal Brisbane \& Womens Hospital. Herston, Australia

Médico epidemiólogo; ${ }^{\mathrm{b}}$ médico cirujano; ${ }^{\mathrm{c}}$ magíster en Epidemiologia Clínica

Recibido: 28-03-14 Aprobado: 06-08-14
}

Citar como: Furuya-Kanamori L, Dulanto-Reinoso CM, Stone JC, Marroquin L, Dulanto-Reinoso VC, Roca JA, et al. Neoplasia escamosa de la superficie ocular en pacientes con pterigion en Perú. Rev Peru Med Exp Salud Publica. 2014;31(4):689-94. 


\section{INTRODUCCIÓN}

La neoplasia escamosa de la superficie ocular (NESO) y el pterigión tienen a la radiación ultravioleta (UV) de la luz solar como factor causal determinante. Además, comparten varios factores de riesgo tales como: sexo masculino, aumento de la edad, tiempo de exposición a la radiación UV (1-3) y la recurrencia de la lesión ${ }^{(4,5)}$. En algunas situaciones puede ser difícil distinguir entre ambas enfermedades, dado que ambas lesiones se localizan en la fisura interpalpebral, principalmente en el limbo afectando a la conjuntiva, córnea, o ambos; lo que podría ocasionar un error diagnóstico ${ }^{(6-8)}$. Esta es una consideración importante dado que el manejo del pterigión y de la NESO son distintos, por lo que requieren de un tratamiento médico especializado y de técnicas quirúrgicas diferentes ${ }^{(9-11)}$. Cabe resaltar que un abordaje quirúrgico inicial inadecuado supone un mayor riesgo para la recurrencia de la lesión neoplásica (12). Asimismo, en este tipo de lesiones neoplásicas es común la metástasis y el uso de tratamientos más agresivos como la enucleación y la exenteración ${ }^{(13,14)}$.

A nivel mundial, el examen histopatológico obligatorio de muestras conjuntivales ha dado lugar a investigaciones que evalúan la frecuencia de NESO no sospechada en especímenes de pterigión ${ }^{(15-18)}$. Específicamente en Perú, no se han realizado estudios de esta naturaleza. La prevalencia de pterigión en la población peruana se estima en $31 \%{ }^{(19)}$, asimismo, en algunas situaciones la NESO puede ser clínicamente indistinguible de un pterigión, por lo tanto, se podría esperar un número alto de especímenes de pterigión con NESO no sospechada. El presente estudio tiene como objetivo estimar la frecuencia de NESO no sospechada en especímenes de pterigión, examinar la precisión del diagnóstico clínico en la identificación de pterigión y NESO y comparar las características demográficas y clínicas de los pacientes con pterigión y NESO.

\section{MATERIALES Y MÉTODOS}

Se examinaron los informes histopatológicos de los pacientes con diagnóstico clínico de pterigión y/o NESO que fueron quirúrgicamente tratados. Todos los procedimientos quirúrgicos se realizaron entre marzo de 2009 y diciembre de 2012 en el Instituto Nacional de Oftalmología (INO), una institución de tercer nivel de atención en Lima, Perú.

Las indicaciones quirúrgicas para el pterigión fueron la presencia de una lesión mayor de $2 \mathrm{~mm}$ en la córnea, disminución de la visión y la preocupación del paciente con respecto a la apariencia estética del ojo. Las escisiones del pterigión fueron realizadas por diferentes cirujanos que utilizaron la técnica de autoinjerto conjuntival. Por otro lado, cuando se sospechó de NESO, se usó una técnica sin contacto dejando 4 a $5 \mathrm{~mm}$ de márgenes, epiteliectomía con alcohol y crioterapia a los márgenes quirúrgicos.

Todas las muestras se fijaron en formol y se procesaron en su totalidad en inclusión de parafina para el examen histopatológico. Las muestras fueron seccionadas y teñidas con hematoxilina-eosina para su análisis. Dos o tres cortes fueron examinados por espécimen. Todas las muestras fueron examinadas por un médico oftalmólogo con subespecialidad en patología y oncología ocular (LM) quien no tuvo acceso al diagnóstico clínico de la muestra enviada. Se recogieron informes histopatológicos de adultos mayores de 18 años de edad. Los datos recogidos incluyeron el sexo del paciente, la edad del paciente al momento del procedimiento quirúrgico, lesión primaria o recurrente, el lado del ojo afectado y la ubicación en el ojo. La medida de resultado en este estudio fue el diagnóstico histopatológico de pterigión (degeneración elastótica del colágeno con la proliferación fibrovascular) o de NESO que incluye displasia leve, displasia moderada, displasia severa, carcinoma in situ y carcinoma de células escamosas. La clasificación histopatológica utilizada para las displasias, carcinomas in situ y carcinomas de células escamosas se basó en el espesor completo de la biopsia conjuntival.

Se calculó la frecuencia no sospechada de NESO en especímenes de pterigión como la proporción de diagnósticos histopatológicos de NESO en todos los especímenes de pterigión clínicamente identificados. Las asociaciones entre las características demográficas y clínicas de los pacientes y el diagnóstico histopatológico se examinaron mediante la prueba de chi-cuadrado. Especímenes con diagnóstico concurrente de NESO y pterigión se juntaron con los casos de NESO con el fin de evaluar la exactitud del diagnóstico clínico, la sensibilidad se calculó como la proporción de los informes de patología con NESO que fueron diagnosticados clínicamente como NESO. La especificidad se calculó como la proporción de los informes de patología de pterigión que fueron diagnosticados clínicamente como pterigión. El cociente de probabilidad positivo (Likelihood ratio, LR+) se calculó como la probabilidad de que un paciente con NESO dada la probabilidad de ser diagnosticados clínicamente con NESO; del mismo modo, el cociente de probabilidad negativo (LR-) se calculó como la probabilidad de que un paciente sin NESO dada la probabilidad de no haber sido diagnosticado con NESO. Los LR fueron elegidos sobre los valores predictivos para indicar la probabilidad 
de presentar NESO después del diagnóstico clínico, ya que la prevalencia en la población de NESO era desconocida. Además, los LR son independientes de la prevalencia de la enfermedad, por lo tanto, se puede aplicar en diferentes poblaciones ${ }^{(20)}$.

Se emplearon modelos de regresión log-log negativo ${ }^{(21)}$ para examinar los factores de riesgo demográficos y clínicos asociados con un aumento de las probabilidades de NESO. Los modelos univariados fueron realizados inicialmente para evaluar de forma independiente el sexo, la edad, la recurrencia de la lesión, el ojo afectado y el lado en que se produjo la lesión. Estas variables se analizaron conjuntamente en un modelo multivariado para el ajuste de los factores de riesgo. El ajuste del modelo estadístico se examinó utilizando el criterio de información Akaike ${ }^{22)}$. El error tipo 1 se mantuvo en el $5 \%$ (alfa $=0,05$ ) para todas las pruebas estadísticas. La odds ratio (OR) y los intervalos de confianza del 95\% (IC 95\%) se presentaron para la regresión log-log negativo. Todos los análisis estadísticos se realizaron utilizando Stata ${ }^{\circ}$, versión 12. (Stata Corporation; College Station, TX).

Debido a que se realizó un estudio retrospectivo utilizando datos previamente recolectados, el consentimiento escrito de los pacientes no se obtuvo; sin embargo, el acceso a las historias clínicas y el análisis de los datos fueron aprobados por el Comité de Ética del INO.

\section{RESULTADOS}

En total, se recogieron 3021 informes histopatológicos que cumplieron con los criterios de inclusión/exclusión. De los pacientes con pterigión 982 (32,5\%) eran varones y el rango de edad fue entre 18 y 98 años (media de 50,4 años, DE 12,9). Las lesiones recurrentes se observaron en $37(1,2 \%)$ pacientes que presentaron una distribución similar del ojo afectado; asimismo, las lesiones del lado nasal ocurrieron en 2736 (90,6\%) pacientes. En los pacientes con NESO, 61 (48\%) eran varones y el rango de edad varió entre 29 y 95 años (media 60,9 años, DE 16,1). Las lesiones recurrentes se presentaron en 6 $(4,7 \%)$ pacientes, fueron observados $71(55,9 \%)$ casos con compromiso del ojo derecho y se evidenció que las lesiones nasales fueron más comunes $(70,1 \%)$.

Hubo diferencias significativas entre los pacientes con pterigión y NESO; la proporción de pacientes del sexo masculino fue mayor en el grupo con NESO $(p<0,001)$; en promedio, los pacientes con NESO eran 10,5 años mayores que los pacientes con pterigión $(p<0,001)$; la recurrencia de la lesión fue cuatro veces mayor en el grupo con NESO $(p<0,001)$ y las lesiones del lado
Tabla 1. Características demográficas y clínicas de los pacientes según diagnostico histopatológico

\begin{tabular}{|c|c|c|c|c|}
\hline & Total & Pterigión & NESO & \\
\hline Características & $\mathbf{N}(\%)$ & $\mathbf{N}(\%)$ & $\mathbf{N}(\%)$ & $p$ \\
\hline Pacientes & $3021(100)$ & $2894(95,8)$ & $127(4,2)$ & \\
\hline \multicolumn{5}{|l|}{ Sexo } \\
\hline Femenino & $2039(67,5)$ & $1973(68,2)$ & $66(52,0)$ & $<0,001$ \\
\hline Masculino & $982(32,5)$ & $921(31,8)$ & $61(48,0)$ & \\
\hline \multicolumn{5}{|l|}{ Edad (en años) } \\
\hline$\leq 40$ & $717(23,7)$ & $704(24,3)$ & $13(10,2)$ & $<0,001$ \\
\hline $41-60$ & $1628(53,9)$ & $1579(54,6)$ & $49(38,6)$ & \\
\hline $61-80$ & $624(20,7)$ & $577(19,9)$ & $47(37,0)$ & \\
\hline$\geq 81$ & $52(1,7)$ & $34(1,2)$ & $18(14,2)$ & \\
\hline \multicolumn{5}{|c|}{ Recurrencia de la lesión } \\
\hline No & $2984(98,8)$ & $2863(98,9)$ & $121(95,3)$ & $<0,001$ \\
\hline Sí & $37(1,2)$ & $31(1,1)$ & $6(4,7)$ & \\
\hline \multicolumn{5}{|l|}{ Ojo } \\
\hline Derecho & $1503(49,8)$ & $1432(49,5)$ & $71(55,9)$ & 0,160 \\
\hline Izquierdo & $1518(50,2)$ & $1462(50,5)$ & $56(44,1)$ & \\
\hline \multicolumn{5}{|l|}{ Lado } \\
\hline Nasal & $2736(90,6)$ & $2647(91,5)$ & $89(70,1)$ & $<0,001$ \\
\hline Temporal & $82(2,7)$ & $47(1,6)$ & $35(27,6)$ & \\
\hline Nasotemporal & $203(6,7)$ & $200(6,9)$ & $3(2,4)$ & \\
\hline
\end{tabular}

NESO: Neoplasia escamosa de la superficie ocular

temporal ocurrieron con más frecuencia en los pacientes con NESO $(p<0,001)$ Tabla 1.

Dos mil ochocientos noventa y un muestras fueron examinadas histopatológicamente con diagnóstico clínico de pterigión, 92 especímenes como NESO y 16 especímenes como pterigión con NESO concurrente. Entre las muestras enviadas como pterigión, 19 recibieron el diagnóstico histopatológico de NESO. La frecuencia insospechada de NESO en especímenes de pterigión fue de $0,7 \%$ (Tabla 2). El diagnóstico clínico de NESO o pterigión con NESO concurrentes ocurrió en $108 / 127$ de los pacientes con patología probada de NESO, mientras que un diagnóstico clínico errado de NESO o pterigión con NESO concurrente ocurrió en 22/2894 de pacientes con patología probada de

Tabla 2. Diagnóstico clínico e histopatológico de los especímenes

\begin{tabular}{lccc}
\hline & \multicolumn{3}{c}{ Diagnóstico histopatológico } \\
\cline { 2 - 4 } Diagnóstico clínico & NESO & Pterigión & Total \\
\hline NESO & 92 & 11 & 103 \\
Pterigión con NESO concurrente & 16 & 11 & 27 \\
Pterigión & 19 & 2872 & 2891 \\
\hline Total & 127 & 2894 & 3021 \\
\hline
\end{tabular}

NESO: neoplasia escamosa de la superficie ocular 
Tabla 3. Precisión diagnóstica del dictamen clínico

\begin{tabular}{lccc}
\hline & \multicolumn{3}{c}{ Diagnóstico histopatológico } \\
\cline { 2 - 4 } Diagnóstico clínico & NESO & Pterigión & Total \\
\hline NESO/Pterigion con NESO & 108 & 22 & 130 \\
concurrente & 19 & 2872 & 2891 \\
Pterigion & 127 & 2894 & 3021 \\
\hline Total &
\end{tabular}

NESO: neoplasia escamosa de la superficie ocular

Sensibilidad: 85,0\% (IC 95\%: 77,4-90,5)

Especificidad: 99,2\% (IC 95\%: 98,8-99,5)

Cociente de probabilidad positivo: 111,8 (IC 95\%: 73,3-170,0)

Cociente de probabilidad negativo: 0,15 (IC 95\%: 0,1-0,2)

pterigión. Por lo tanto, el diagnóstico clínico mostró una sensibilidad del $85,0 \%$ y una especificidad del $99,2 \%$ en la identificación de casos de NESO. Además, con un diagnóstico clínico de NESO existen 111,89 veces más probabilidades de ser diagnosticados correctamente y 0,15 veces menor probabilidades de ser diagnosticados clínicamente con un pterigión (Tabla 3).

Los modelos no ajustados de regresión log-log negativos revelaron que en comparación con las mujeres, los hombres tenían 1,23 mayor probabilidades de desarrollar NESO (IC 95\%: 1,10-1,38). Las probabilidades de NESO incrementaron con el aumento de la edad en comparación con la categoría de edad de referencia (menores de 40 años) de tal manera que aquellos pacientes que tenían entre 61 a 80 años y mayores de 81 años tenían un riesgo significativamente mayor de desarrollar NESO. Los pacientes con una lesión recurrente tuvieron un aumento en las probabilidades de NESO en comparación con aquellos con una lesión primaria (OR 1,76; IC 95\%: 1,17-2,65). Los pacientes con una lesión temporal (OR 4,02; IC 95\%: 2,98-5,43) tuvieron mayor probabilidades de presentar NESO en comparación con una lesión unilateral nasal (Tabla 4).

En el modelo multivariado las probabilidades de NESO para los hombres no disminuyeron sustancialmente del modelo sin ajustar y se mantuvo significativa (OR 1,15; IC 95\%: 1,01-1,30) en comparación con las mujeres. Las probabilidades de NESO para pacientes de 81 años o mayores se redujeron considerablemente $(>10 \%)$ en comparación con el modelo no ajustado, aunque se mantuvo significativa (OR 3,10; IC 95\%: 2,09-4,58) en comparación con los pacientes menores de 40 años. Las probabilidades de lesiones recurrentes no se redujeron sustancialmente $(<10 \%)$ del modelo univariado y se mantuvo significativa. Lesiones del lado temporal presentaron un aumento significativo de la probabilidad de desarrollar NESO en comparación con lesiones del lado nasal cuando es ajustado con otras variables estudiadas (Tabla 4).
Tabla 4. Modelos de regresión para la asociación entre las características demográficas y clínicas y la presencia de neoplasia escamosa de la superficie ocular

\begin{tabular}{|c|c|c|c|c|}
\hline \multirow[b]{2}{*}{ Características } & \multicolumn{2}{|c|}{$\begin{array}{l}\text { Modelos sin } \\
\text { ajustar }\end{array}$} & \multicolumn{2}{|c|}{$\begin{array}{l}\text { Modelo } \\
\text { ajustado* }\end{array}$} \\
\hline & OR & (IC 95\%) & OR & (IC 95\%) \\
\hline \multicolumn{5}{|l|}{ Sexo } \\
\hline Femenino & 1,00 & & 1,00 & \\
\hline Masculino & 1,23 & $(1,10-1,38)$ & 1,15 & $(1,01-1,30)$ \\
\hline \multicolumn{5}{|l|}{ Edad (en años) } \\
\hline$\leq 40$ & 1,00 & & 1,00 & \\
\hline $41-60$ & 1,14 & $(0,98-1,34)$ & 1,14 & $(0,97-1,35)$ \\
\hline $61-80$ & 1,55 & $(1,31-1,84)$ & 1,54 & $(1,28-1,85)$ \\
\hline$\geq 81$ & 3,78 & $(2,59-5,51)$ & 3,10 & $(2,09-4,58)$ \\
\hline \multicolumn{5}{|c|}{ Recurrencia de la lesión } \\
\hline No & 1,00 & & 1,00 & \\
\hline Sí & 1,76 & $(1,17-2,65)$ & 1,59 & $(1,03-2,46)$ \\
\hline \multicolumn{5}{|c|}{ 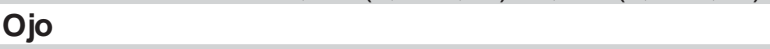 } \\
\hline Derecho & 1,00 & & 1,00 & \\
\hline Izquierdo & 0,93 & $(0,83-1,03)$ & 0,92 & $(0,81-1,03)$ \\
\hline \multicolumn{5}{|l|}{ Lado } \\
\hline Nasal & 1,00 & & 1,00 & \\
\hline Temporal & 4,02 & $(2,98-5,43)$ & 3,57 & $(2,63-4,85)$ \\
\hline Nasotemporal & 0,81 & $(0,62-1,07)$ & 0,77 & $(0,58-1,05)$ \\
\hline
\end{tabular}

* Ajustado para sexo, edad, recurrencia de la lesión, ojo y lado de la lesión.

\section{DISCUSIÓN}

Los hallazgos del presente estudio indican que la frecuencia de NESO no sospechada en especímenes de pterigión en esta población peruana es del 0,65\%. Esta frecuencia menor del $2 \%$ es similar a la encontrada en Tailandia ${ }^{(18)}$, EE.UU. ${ }^{\left({ }^{(8)}\right.}$ y Canadá ${ }^{(17)}$. Por el contrario, dos estudios realizados en Australia encontraron frecuencias no sospechada del $5^{\left({ }^{(16)}\right.}$ y $9,8 \%{ }^{(15)}$. Es probable que estas investigaciones australianas reportaran frecuencias más altas, porque el estudio histopatológico de los especímenes de pterigión se desarrolló específicamente para identificar la presencia de NESO. Otras razones que pueden explicar la mayor frecuencia en Australia incluyen un índice de radiación UV alto debido a la deficiencia de la capa de ozono en esta región (23,24), la mayor susceptibilidad de la raza caucásica para desarrollar NESO que está relacionada a la piel blanca y/o iris pálido ${ }^{(23)}$ y posiblemente a factores genéticos ${ }^{(8,15-}$ $17,25)$.

El diagnóstico clínico demostró ser altamente específico para la detección de NESO o pterigión con NESO concurrente, por lo tanto, el diagnóstico clínico generalmente identificará la presencia de la enfermedad. Sin embargo, se encontró que la sensibilidad solo era moderada. Aunque el diagnóstico clínico identifica a la mayoría de los pacientes con NESO, un diagnóstico 
clínico negativo de NESO o pterigión con NESO concurrente no descarta por completo la posibilidad de NESO no sospechada. Dado un diagnóstico clínico de NESO (LR+), la probabilidad de presentarlo aumenta drásticamente, por lo que es necesario desarrollar un tratamiento médico y un enfoque quirúrgico específico. Sin embargo, dado un diagnóstico clínico de no presentar NESO (LR-), la probabilidad de presentarlo disminuye moderadamente y la posibilidad de NESO no sospechada permanece (20). En el INO, cuando un oftalmólogo evalúa a un paciente con pterigión de características clínicas atípicas lo refiere al Servicio de Oncología Ocular para profundizar su estudio y, por ende, se brinde el tratamiento adecuado.

Al examinar los factores de riesgo demográfico y clínico, se encontró que los pacientes mayores de 61 años tenían mayores probabilidades de desarrollar NESO. Estos hallazgos apoyan estudios previos realizados por Oellers et al. ${ }^{(8)}$ que indican que la NESO ocurre con más frecuencia en pacientes de 60 a 69 años debido a que el envejecimiento es un factor de riesgo significativo para el desarrollo de lesiones neoplásicas malignas ${ }^{(26)}$. Además, la incidencia de cáncer en general aumenta con la edad ${ }^{(27)}$. Esto puede ser atribuido a la duración de la carcinogénesis, al aumento de la susceptibilidad de tejidos más viejos a agentes carcinógenos en etapas tardías y a los efectos sistémicos del envejecimiento, incluyendo la senescencia inmune y al aumento de la producción de citoquinas (28). Los resultados de este estudio revelan que los hombres presentan un aumento de las probabilidades de desarrollar NESO. Las investigaciones de Lee y Hirst ${ }^{(29)}$ y Kao et al. ${ }^{(30)}$ encontraron que el sexo masculino es más susceptibles a presentar NESO. Esto no debe sorprender, teniendo en cuenta que los hombres que residen en Perú a menudo laboran en exteriores, lo cual ocasiona una mayor exposición al medioambiente y a la radiación UV en comparación con las mujeres ${ }^{(31)}$.

Las personas con lesiones temporales tienen un mayor riesgo de desarrollar NESO en comparación con aquellas que presentan una lesión nasal unilateral. Esto apoya la investigación realizada por Kao et al. (30) quienes identificaron que las lesiones localizadas en la conjuntiva temporal se asociaron con mayor grado de NESO, en comparación con lesiones del lado nasal. En contraste, Oellers et al. (8) observaron que las lesiones localizadas en la conjuntiva inferior resultaron ser más frecuentes en pacientes con NESO. Por otra parte, las personas con lesiones recurrentes tuvieron un aumento de las probabilidades de NESO en comparación con los que tenían lesiones primarias. Hallazgos similares se han documentado en la literatura que indican que la recurrencia tras un año de seguimiento varió de 11 a $27 \%{ }^{(8,13)}$. Esto puede explicarse porque un diagnóstico erróneo inicial ocasiona un tratamiento quirúrgico inadecuado que origina la recurrencia de la lesión neoplásica.

Este estudio evidencia la baja frecuencia de NESO no sospechada en especímenes de pterigión en una población peruana y es probable una subestimación de la frecuencia real. Recomendamos que todos los especímenes de pterigión sean enviados de forma rutinaria para su estudio histopatológico con la finalidad de excluir la presencia de NESO, por tal motivo, es necesario realizar estudios que evalúen el costoefectividad de esta intervención. En particular, las lesiones del lado temporal y/o recurrente en personas del sexo masculino y de edad avanzada deben ser examinadas debido al alto riesgo que presentan de desarrollar la enfermedad. Es imprescindible un diagnóstico correcto de pterigión o NESO con la finalidad de proporcionar un tratamiento adecuado, ya que el manejo de estas dos enfermedades es muy diferente.

\section{Fuentes de financiamiento: autofinanciado}

Conflictos de interés: los autores declaran no tener conflictos de interés.

Contribuciones de autoría: LFK participó en la obtención de datos, análisis e interpretación de datos y redacción del artículo. CMDR participó en la obtención de datos de redacción del artículo. JCS participó en el análisis e interpretación de datos y redacción del artículo. LM participó en la concepción y diseño del artículo y el aporte de pacientes o materiales de estudio. VchDR participó en la obtención de datos y redacción del artículo. JAR, FC y GAL participaron en la redacción y revisión crítica del artículo. Todos los autores aprobaron la versión final del artículo. 


\section{REFERENCIAS BIBLIOGRÁFICAS}

1. Mackenzie FD, Hirst LW, Battistutta D, Green A. Risk analysis in the development of pterygia. Ophthalmology. 1992 Jul;99(7):105661.

2. Lee GA, Williams G, Hirst LW, Green AC. Risk factors in the development of ocular surface epithelial dysplasia. Ophthalmology. 1994Feb;101(2):3604.

3. Lee GA, Hirst LW. Ocular surface squamous neoplasia. Surv Ophthalmol. 1995 May-Jun;39(6):429-50.

4. Tabin G, Levin S, Snibson G, Loughnan $\mathrm{M}$, Taylor H. Late recurrences and the necessity for long-term follow-up in corneal and conjunctival intraepithelial neoplasia. Ophthalmology. 1997 Mar;104(3):485-92.

5. Varssano D, Shalev H, Lazar M, Fischer N.Pterygium excision with conjunctival autograft: true survival rate statistics. Cornea. 2013 Sep;32(9):1243-50. doi: 10.1097/ICO.0b013e31828ce09c.

6. McKelvie PA, Daniell M. Impression cytology following mitomycin C therapy for ocular surface squamous neoplasia. Br J Ophthalmol. 2001 Sep;85(9):1115-9.

7. Hamam R, Bhat P, Foster CS. Conjunctival/corneal intraepithelial neoplasia. Int Ophthalmol Clin. 2009 Winter;49(1):63-70. doi: 10.1097/ IIO.0b013e3181924ec3.

8. Oellers P, Karp CL, Sheth A, Kao AA, Abdelaziz A, Matthews JL, et al. Prevalence, treatment and outcomes of coexistent ocular surface squamous neoplasia and pterygium. Ophthalmology. 2013 Mar;120(3):445-50. doi: 10.1016/j. ophtha.2012.08.010.

9. Hirst LW. The treatment of pterygium. Surv Ophthalmol. 2003 MarApr;48(2):145-80.

10. Basti S, Macsai MS. Ocular surface squamous neoplasia: a review. Cornea. 2003 Oct;22(7):687-704.

11. Ang LP, Chua JL, Tan DT. Current concepts and techniques in pterygium treatment. Curr Opin Ophthalmol. 2007 Jul;18(4):308-13.

12. Yousef YA, Finger PT. Squamous carcinoma and dysplasia of the conjunctiva and cornea: an analysis of 101 cases. Ophthalmology. 2012 Feb;119(2):233-40. doi: 10.1016/j. ophtha.2011.08.005.

13. McKelvie PA, Daniell M, McNab A, Loughnan M, Santamaria JD. Squamous cell carcinoma of the conjunctiva: a series of 26 cases. $\mathrm{Br} \mathrm{J}$ Ophthalmol. 2002 Feb;86(2):168-73.

14. Babar TF, Khan MN, Hussain M, Shah SA, Khan MY, Khan MD. Spectrum of ocular surface squamous neoplasia. J Coll Physicians Surg Pak. 2007 Jun;17(6):344-6.

15. Hirst LW, Axelsen RA, Schwab I. Pterygium and associated ocular surface squamous neoplasia. Arch Ophthalmol. 2009 Jan;127(1):31-2. doi: 10.1001/ archophthalmol.2008.531.

16. Chui J, Coroneo MT, Tat LT, Crouch $\mathrm{R}$, Wakefield D, Di Girolamo N. Ophthalmic pterygium: a stem cell disorder with premalignant features. Am J Pathol. 2011 Feb;178(2):817-27. doi: 10.1016/j.ajpath.2010.10.037.

17. Yeung SN, Kim P, Lichtinger A, Amiran MD, Cote E, Teitel S, et al. Incidence of ocular surface squamous neoplasia in pterygium specimens: an 8-year survey. Br J Ophthalmol. 2011 Apr;95(4):592. doi: 10.1136/bjo.2010.197491.

18. Artornsombudh P, Sanpavat A, Tinnungwattana U, Tongkhomsai V, Sansopha L, Tulvatana W. Prevalence and clinicopathologic findings of conjunctival epithelial neoplasia in pterygia. Ophthalmology. 2013 Jul;120(7):133740. doi: 10.1016/j.ophtha.2012.12.020.

19. Rojas JR, Malaga H. Pterygium in Lima, Peru. Ann Ophthalmol. 1986 Apr;18(4):147-9.

20. Doi S. Understanding evidence in health care: using clinical epidemiology. South Yarra, VIC: Palgrave Macmillan; 2012.

21. Dobson AJ. An Introduction to Generalized Linear Models. 2da ed. Florida: Chapman \& Hall/CRC; 2010.

22. Akaike H. A new look at the statistical model identification. IEEE Trans Autom Control. 1974;19(6):716-23.

23. Yam JC, Kwok AK. Ultraviolet light and ocular diseases. Int Ophthalmol. 2014 Apr;34(2):383-400. doi: 10.1007/s10792-013-9791-x.
24. Karcioglu ZA, Wagoner MD. Demographics, etiology, and behavior of conjunctival squamouscell carcinoma in the 21st century. Ophthalmology. 2009 Nov;116(11):2045-6. doi: 10.1016/j.ophtha.2009.09.031.

25. Tong L, Chew J, Yang H, Ang LP, Tan DT, Beuerman RW. Distinct gene subsets in pterygia formation and recurrence: dissecting complex biological phenomenon using genome wide expression data. BMC Medical Genomics. 2009;2:14.

26. Denduluri N, Ershler WB. Aging biology and cancer. Sem Oncol. 2004;31(2):137-48.

27. Yancik R, Ries LA. Cancer in older persons: an international issue in an aging world. Semin Oncol. 2004 Apr;31(2):128-36.

28. Balducci L, Beghe C. The application of the principles of geriatrics to the management of the older person with cancer. Crit Rev Oncol Hematol. 2000 Sep;35(3):147-54.

29. Lee GA, Hirst LW. Retrospective study of ocular surface squamous neoplasia. Aust N Z J Ophthalmol. 1997 Nov;25(4):269-76.

30. Kao AA, Galor A, Karp CL, Abdelaziz A, Feuer WJ, Dubovy SR. Clinicopathologic correlation of ocular surface squamous neoplasms at Bascom Palmer Eye Institute: 2001 to 2010. Ophthalmology. 2012 Sep;119(9):1773-6. doi: 10.1016/j. ophtha.2012.02.049.

31. Reinau D, Weiss M, Meier CR, Diepgen TL, Surber C. Outdoor workers' sun-related knowledge, attitudes, and protective behaviours: A systematic review of cross-sectional and interventional studies. Br J Dermatol. 2013 May;168(5):928-40. doi: 10.1111/bjd.12160.

Correspondencia: Carlos Dulanto Reinoso Dirección: Instituto Nacional de Oftalmología. Av. Tingo María 398 Breña, Lima 05, Perú Teléfono: 5112264894

Correo electrónico: carlos.dulanto.r@upch.pe 